\title{
Predictive Factors for Severe Outcomes in Ischemic Colitis
}

\author{
Seok Reyol Choi*, Sam Ryong Jee ${ }^{\dagger}$, Geun Am Song ${ }^{\ddagger}$, Seun Ja Park ${ }^{\S}$, Jong Hun Lee", Chul Soo Song", and Hee Ug Park \\ ${ }^{*}$ Department of Internal Medicine, Busan Hangun Hospital, ${ }^{\dagger}$ Department of Internal Medicine, Inje University College of Medicine, ${ }^{\ddagger}$ Department \\ of Internal Medicine, Pusan National University School of Medicine, ${ }^{8}$ Department of Internal Medicine, Kosin University Gospel Hospital, \\ "Department of Internal Medicine, Dong-A University College of Medicine, "Department of Internal Medicine, Good Samsun Hospital, and \\ \# Department of Internal Medicine, Maryknoll Medical Center, Busan, Korea
}

Background/Aims: Ischemic colitis includes a wide clinical spectrum ranging from mild to severe forms. This study aimed to determine the factors that are related to the occurrence of severe ischemic colitis. Methods: This multicenter study was conducted retrospectively in Korea. The patients were divided into mild and severe groups. This study surveyed clinical characteristics, blood tests, endoscopic findings, and imaging studies. Results: In the comparison of comorbidities, the severe group had a higher ratio of chronic kidney disease than the mild group ( $p=0.001$ ). In the blood test, the severe group had a reduced number of platelets $(p=0.018)$ and a higher $C$-reactive protein value $(p=0.001)$. The severe group had a higher ratio of involvement of the right colon $(p=0.026)$. The Eastern Cooperative Oncology Group (ECOG) performance status score of the patients showed that the severe group had higher scores than the mild group ( $p=0.003$ ). A multivariate analysis showed that chronic kidney disease and high ECOG performance status scores were significant risk factors. Conclusions: If patients diagnosed with ischemic colitis are also treated for chronic kidney disease or have poor performance status, more attention and early intervention are necessary. (Gut Liver 2015;9:761-766)

Key Words: Colitis, ischemic; Predictive factors; Outcome

\section{INTRODUCTION}

Ischemic colitis is a vascular disease caused by insufficient blood supply to the colon, thereby causing inflammation in the colon. It is the most frequent form of ischemic bowel disease. ${ }^{1}$ The occurrence of ischemic colitis increases with age in general; having said that, it can occur at any age. It has been known that this disease is accompanied by various diseases such as hypertension, coronary heart disease, diabetes, chronic obstructive pulmonary disease, and chronic kidney disease. ${ }^{2}$

The clinical features vary so that some patients do not even need medical treatment owing to natural improvement within 2 to 3 days in the case of local invasion to mucosa or submucosa, whereas others may die if inflammation and necrosis are accompanied over the entire wall of the colon.,

In general, ischemic colitis can be recovered with conservative treatment only; however, in some cases, surgical treatment is needed; in extreme cases, it may result in death. Therefore, it is highly important to treat patients differently by recognizing the factors that influence bad effects prognostically.

In previous studies, the following poor prognostic factors have been found: old age, showing hemodynamic instability at an early stage of ischemic colitis, continued colon paralysis, involvement of right colon, medical history of hypertension and malignant tumors, and end-stage renal disease with hemodialysis. However, these factors have differed across studies. ${ }^{5-9}$

Therefore, the authors aimed to determine the factors that are related to the occurrence of severe ischemic colitis in order to provide faster and more active treatment for patients.

\section{MATERIALS AND METHODS}

\section{Patients}

A retrospective study was undertaken of the records of patients who were diagnosed with ischemic colitis in two secondary hospitals (Maryknoll Medical Center and Good Samsun Hospital) and four university hospitals (Dong-A University, Kosin University, Pusan National University, and Inje University)

\footnotetext{
Correspondence to: Sam Ryong Jee

Department of Gastroenterology, Inje University Busan Paik Hospital, Inje University College of Medicine, 75 Bokji-ro, Busanjin-gu, Busan 47392, Korea

Tel: +82-51-890-6536, Fax: +82-51-892-0273, E-mail: srjee@inje.ac.kr

Received on April 7, 2015. Revised on May 12, 2015. Accepted on May 19, 2014. Published online September 9, 2015

pISSN 1976-2283 eISSN 2005-1212 http://dx.doi.org/10.5009/gnl15167

(a) This is an Open Access article distributed under the terms of the Creative Commons Attribution Non-Commercial License (http://creativecommons.org/licenses/by-nc/4.0) which permits unrestricted non-commercial use, distribution, and reproduction in any medium, provided the original work is properly cited.
} 
located in Busan, Korea, from January 2000 to December 2011. This study chose patients who were confirmed via the findings in the colonoscopy or diagnosed with ischemic colitis via pathological examination after a colonoscopic evaluation of the entire colon. The colonoscopy findings were based on the presence of petechiae, pale mucous membranes, congestion, ulcers, and exudate whereas pathological examination findings were judged by pathology specialists based on the presence of removal and regeneration of the mucosal epithelium, coagulation necrosis in the mucous membranes, bleeding in the lamina propria, invasion of inflammatory cells in the lamina propria, crypt necrosis or loss, and fibrous tissue proliferation. Patients were divided into mild and severe groups. Patients in the mild group were defined as those who improved with only conservative treatment, whereas patients in the severe group were defined as those who needed surgical treatments or died.

\section{Methods}

Inclusion criteria included (1) a colonoscopic or surgical evaluation of the entire colon and (2) a pathologic report that described signs of pathognomonic or consistent with the diagnosis of ischemic colitis. Patients were excluded if they had prior colonic operation, bowel obstruction, colonic or pelvic cancer, Crohn's disease or ulcerative colitis.

Patients in the mild and severe groups were surveyed to find the following information: age, gender, symptoms, comorbidities, blood test, endoscopic findings, abdominal computed tomography (CT) scan and angiography findings, medical records about Eastern Cooperative Oncology Group (ECOG) performance status. ${ }^{10}$ The symptoms were judged by the presence of abdominal pain and bloody stools whereas comorbidities were defined as hypertension, diabetes, ischemic heart disease, cerebrovascular disorder, cardiac failure, arrhythmia, peripheral vascular disease, thyroid disease, chronic obstructive pulmonary disease, chronic kidney disease, presence of hemodialysis or peritoneal dialysis, irritable bowel syndrome, and hyperlipidemia as well as total cholesterol, triglyceride, low-density lipoprotein, high-density lipoprotein, total protein, albumin, hemoglobin, white blood cell, platelet, erythrocyte sedimentation rate, and C-reactive protein (CRP). In the endoscopic findings, the colon was divided into four sections: right colon (from appendix to immediately before the hepatic flexure), left colon (from rectum to splenic flexure), transverse colon (hepatic flexure to immediately before the splenic flexure), and whole colon. Patients had abdominal CT and angiography to determine abnormal superior mesenteric and inferior mesenteric arteries.

\section{Statistical analysis}

SPSS software version 16.0 (SPSS Inc., Chicago, IL, USA) was used for the statistical analysis. To compare the mild and severe groups, a t-test was used to compare continuous variables and a chi-square test was used to compare categorical variables. In order to verify the prognostic factors that are related to the occurrence of severe ischemic colitis, logistic regression analysis was conducted and the statistical significance was set to $p$-value of $<0.05$.

\section{RESULTS}

The study subjects were 292 patients from four university hospitals and two secondary hospitals. Among them, the mild group had 259 patients and the severe group, 33 patients (Table 1). Among 33 cases of severe group, 27 cases were from university hospitals and six from secondary hospitals. Among patients in the severe group, five had surgical treatment and 28 died later. The mean age of the patients was $65.83 \pm 14.50$ years. The number of male and female patients was 115 and 177, respectively. A comparison of the clinical characteristics between mild and severe groups showed no significant difference in age, gender, and symptoms. A comparison of the comorbidities between the two groups showed no significant difference in the ratio of high blood pressure, diabetes, cardiovascular and cerebrovascular diseases, arrhythmia, peripheral vascular disease, thyroid disease, chronic obstructive pulmonary disease, irritable bowel syndrome, and hyperlipidemia; however, the ratio of chronic kidney disease with hemodialysis was significantly higher in the severe group than in the mild group ( $p=0.001$ ). In the blood test, the severe group showed less number of platelets $(p=0.018)$ and higher CRP value ( $p=0.001$ ), but the other categories showed no significant difference between the two groups (Table 1).

In the colonoscopy findings, the occurrence ratio of mild ischemic colitis was $16.98 \%, 62.93 \%, 5.02 \%$, and $15.07 \%$ in the right, left, transverse, and whole colon, respectively, whereas that of severe ischemic colitis was 30.30\%, 42.42\%, 6.06\%, and $21.22 \%$, respectively, which showed no statistical significance (Table 2). However, in the comparison for the right colon, the severe group had a significantly higher ratio of invasion to the right colon than the mild group (51.52\% vs $32.05 \%, \mathrm{p}=0.026$ ) (Table 2). There was no significant difference between the two groups in relation to the presence of lesions in the superior mesenteric artery and inferior mesenteric artery as determined via abdominal CT and angiography. The ECOG performance status score of the patients showed that the severe group had higher scores than the mild group ( $\mathrm{p}=0.003$ ).

The which aimed to determine prognostic factors that influence severe ischemic colitis, showed that chronic kidney disease (odds ratio [OR], 5.289; 95\% confidence interval [CI], 1.31 to 21.38; $p=0.019)$ and high ECOG performance status score (OR, $1.690 ; 95 \% \mathrm{CI}, 1.11$ to $2.58 ; \mathrm{p}=0.015)$ were significant risk factors (Table 3). In the univariate analysis, involving the right colon, hemodialysis, number of platelets, and CRP value had statistical significance, but in the multivariate analysis, they 
Table 1. Baseline Characteristics of Patients with Ischemic Colitis

\begin{tabular}{|c|c|c|c|}
\hline Variable & Mild ( $n=259)$ & Severe $(n=33)$ & p-value \\
\hline Age, yr & $67.39 \pm 13.53$ & $65.41 \pm 14.72$ & 0.164 \\
\hline Male sex & $97 / 259(37.45)$ & $18 / 33(54.55)$ & 0.058 \\
\hline \multicolumn{4}{|l|}{ Symptom } \\
\hline Abdominal pain & 203/259 (78.38) & $27 / 33(81.82)$ & 0.735 \\
\hline Hematochezia & $187 / 259(72.20)$ & $22 / 33(66.67)$ & 0.441 \\
\hline \multicolumn{4}{|l|}{ Comorbidities } \\
\hline Hypertension & $118 / 259(45.56)$ & $20 / 33(60.61)$ & 0.103 \\
\hline $\mathrm{DM}$ & $54 / 259(20.85)$ & 7/33 (21.21) & 0.962 \\
\hline IHD & $31 / 259$ (11.97) & $5 / 33(15.15)$ & 0.600 \\
\hline Stroke & 20/259 (7.72) & 3/33 (9.09) & 0.783 \\
\hline $\mathrm{CHF}$ & $17 / 259(6.56)$ & $5 / 33(15.15)$ & 0.078 \\
\hline Arrhythmia & 23/259 (8.88) & $5 / 33(15.15)$ & 0.249 \\
\hline PVD & $6 / 259(2.32)$ & $2 / 33(6.06)$ & 0.215 \\
\hline Hyperthyroidism & $2 / 259(0.78)$ & $0 / 33$ & 0.612 \\
\hline Hypothyroidism & $7 / 259(2.70)$ & $0 / 33$ & 0.339 \\
\hline COPD & $6 / 259(2.31)$ & $2 / 33(6.06)$ & 0.215 \\
\hline CKD (stage V) & $14 / 259(5.41)$ & $11 / 33(33.33)$ & 0.001 \\
\hline $\mathrm{HD}$ & $5 / 259(1.93)$ & $5 / 33(15.15)$ & 0.001 \\
\hline PD & $1 / 259(0.39)$ & $0 / 33$ & 0.612 \\
\hline IBS & $11 / 259(4.25)$ & $0 / 33$ & 0.228 \\
\hline Hyperlipidemia & $26 / 259(10.04)$ & $4 / 33(12.12)$ & 0.711 \\
\hline \multicolumn{4}{|l|}{ Laboratory findings } \\
\hline Total cholesterol, mg/dL & $141.39 \pm 55.40$ & $155.96 \pm 45.42$ & 0.281 \\
\hline $\mathrm{TG}, \mathrm{mg} / \mathrm{dL}$ & $136.25 \pm 107.08$ & $107.51 \pm 73.42$ & 0.309 \\
\hline $\mathrm{LDL}, \mathrm{mg} / \mathrm{dL}$ & $80.47 \pm 41.59$ & $86.33 \pm 36.66$ & 0.603 \\
\hline $\mathrm{HDL}, \mathrm{mg} / \mathrm{dL}$ & $37.73 \pm 12.51$ & $44.55 \pm 25.10$ & 0.519 \\
\hline Total protein, g/dL & $6.15 \pm 1.28$ & $6.42 \pm 0.98$ & 0.257 \\
\hline Albumin, g/dL & $3.42 \pm 0.79$ & $3.60 \pm 0.67$ & 0.070 \\
\hline $\mathrm{Hb}, \mathrm{g} / \mathrm{dL}$ & $11.33 \pm 1.76$ & $12.15 \pm 2.32$ & 0.203 \\
\hline $\mathrm{WBC}, / \mu \mathrm{L}$ & $11,045.12 \pm 5,295.36$ & $11,653.51 \pm 9,217.89$ & 0.780 \\
\hline $\mathrm{PLT}, / \mathrm{mm}^{3}$ & $232.33 \pm 92.03$ & $191.06 \pm 104.16$ & 0.018 \\
\hline $\mathrm{ESR}, \mathrm{mm} / \mathrm{hr}$ & $30.23 \pm 24.76$ & $40.17 \pm 31.54$ & 0.125 \\
\hline $\mathrm{CRP}, \mathrm{mg} / \mathrm{dL}$ & $5.59 \pm 7.02$ & $11.70 \pm 12.14$ & 0.001 \\
\hline ECOG performance status score & & & 0.003 \\
\hline 0 & $104 / 259(40.15)$ & $5 / 33(15.15)$ & \\
\hline 1 & $103 / 259$ (39.77) & $12 / 33(36.36)$ & \\
\hline 2 & $35 / 259(13.51)$ & $12 / 33(36.36)$ & \\
\hline 3 & $13 / 259(5.02)$ & 3/33 (9.09) & \\
\hline 4 & $4 / 259(1.55)$ & $1 / 33(3.04)$ & \\
\hline
\end{tabular}

Data are presented as mean \pm SD or number $(\%)$.

$\mathrm{DM}$, diabetes mellitus; IHD, ischemic heart disease; CHF, congestive heart failure; PVD, peripheral vascular disease; COPD, chronic obstructive pulmonary disease; CKD, chronic kidney disease; HD, hemodialysis; PD, peritoneal dialysis; IBS, irritable bowel syndrome; TG, triglyceride; LDL, lowdensity lipoprotein; HDL, high-density lipoprotein; Hb, hemoglobin; WBC, white blood cell; PLT, platelet; ESR, erythrocyte sedimentation rate; CRP, C-reactive protein; ECOG, Eastern Cooperative Oncology Group. 
Table 2. Location of Ischemic Colitis

\begin{tabular}{|c|c|c|c|}
\hline Variable & Mild ( $n=259)$ & Severe $(n=33)$ & p-value \\
\hline Location & & & 0.682 \\
\hline Right colon & $44(16.98)$ & $10(30.30)$ & \\
\hline Left colon & $163(62.93)$ & $14(42.42)$ & \\
\hline Transverse colon & $13(5.02)$ & $2(6.06)$ & \\
\hline Whole colon & $39(15.07)$ & $7(21.22)$ & \\
\hline Right colon vs all others & & & 0.026 \\
\hline Involving right colon & $83 / 259$ (32.05) & $17 / 33(51.52)$ & \\
\hline Noninvolving right colon & $176 / 259(67.95)$ & $16 / 33(48.48)$ & \\
\hline Abdominal CT & $190 / 259(73.36)$ & $23 / 33(69.69)$ & 0.656 \\
\hline SMA stenosis & $14 / 190$ (7.37) & $3 / 23(13.04)$ & 0.513 \\
\hline IMA stenosis & $5 / 190(2.63)$ & $0 / 23$ & 0.597 \\
\hline Angiography & $13 / 259(5.02)$ & $1 / 33(3.03)$ & 0.535 \\
\hline SMA stenosis & $3 / 13(23.08)$ & $0 / 1$ & 0.521 \\
\hline IMA stenosis & $10 / 13(76.92)$ & $1 / 1(100.0)$ & 0.540 \\
\hline
\end{tabular}

Data are presented as number (\%).

CT, computed tomography; SMA, superior mesenteric artery; IMA, inferior mesenteric artery.

Table 3. Multivariate Analysis of Predictive Factors for Severe Ischemic Colitis

\begin{tabular}{llcc}
\hline \multicolumn{1}{c}{ Variable } & \multicolumn{1}{c}{$\mathrm{CI}$} & $\mathrm{OR}$ & $\mathrm{p}$-value \\
\hline Location & $0.584-3.604$ & 1.450 & 0.424 \\
CKD stage V & $1.308-21.378$ & 5.289 & 0.019 \\
HD & $0.316-16.134$ & 2.258 & 0.417 \\
PLT & $0.992-1.003$ & 0.997 & 0.344 \\
CRP & $0.986-1.088$ & 1.036 & 0.160 \\
ECOG & $1.108-2.579$ & 1.690 & 0.015 \\
\hline
\end{tabular}

CI, confidence interval; OR, odds ratio; $\mathrm{CKD}$, chronic kidney disease; HD, hemodialysis; PLT, platelet; CRP, C-reactive protein; ECOG, Eastern Cooperative Oncology Group.

were not found as independent risk factors that were related to the occurrence of severe ischemic colitis.

\section{DISCUSSION}

Ischemic colitis is the most common disease among ischemic diseases that occur in the gastrointestinal tract, and it has no specific symptoms. Colonoscopy is the procedure of choice to establish the diagnosis of colonic ischemia. Barium enema and a CT scan can be useful and angiography has a limited role. Therefore, careful diagnosis is required to correctly diagnose ischemic colitis under such circumstances. Recently, owing to the increase in aging population, chronic cardiopulmonary disease, and prevalence of diabetes, the prevalence of ischemic colitis has increased as well. Most cases recover naturally without conservative treatment, and therefore, the actual prevalence rate seems higher than the known prevalence rate. ${ }^{1}$ The clinical features of ischemic colitis vary so that some patients recover with conservative treatment only, whereas others may need surgical treatment owing to extensive necrosis over the whole colon. $^{11}$

The mortality rate of our study was $9.6 \%$. This result is consistent with a previous studies, which showed $9.4 \%$ to $17.3 \%$. $^{3,12-14}$ In a systematic discussion with respect to 11 papers that studied 1,049 patients with ischemic colitis, $80.3 \%$ of patients received the conservative treatment whereas $6.2 \%$ of patients died. On the other hand, 39.3\% of patients died after surgical treatment (19.6\%) was received. ${ }^{15}$ Therefore, it is essential to set up a rapid treatment plan by identifying poor prognostic factors in relation to severe ischemic colitis that requires surgical treatment or presents the risk of death.

Currently known prognostic factors of severe ischemic colitis are old age, showing hemodynamic instability at an early stage of ischemic colitis, continued colon paralysis, involvement of right colon, medical history of hypertension and malignant tumors, and end-stage renal disease with hemodialysis. ${ }^{5-9}$ In particular, involvement of the right colon has been known to be related to poor prognosis of ischemic colitis in a number of study results ${ }^{12-14,16}$ owing to decreased blood flow in the superior mesenteric artery in the right colon creating more severe clinical manifestations by extensive ischemic injury in the colon. In this study, the ratio of the involvement of the right colon in the severe group was statistically significant; however, multivariate analysis did not show it to be an independent factor that can influence the severity. As in previous studies, this study also found that chronic kidney disease and hemodialysis showed poor prognosis of ischemic colitis, ${ }^{9,17-19}$ whereas this study showed chronic kidney disease only was verified as an 
independent risk factor in the multivariate analysis. Patients with chronic kidney disease have severe ischemic colitis because of continuous thrombus generation owing to dialysis and repetitive hemodynamic changes as two of the major causes. In hemodialysis patients, a decrease in blood amount, rapid movement of body fluids, and the occurrence of hypotension can cause the contraction of the superior mesenteric artery, thereby inducing severe ischemic colitis. ${ }^{13}$ In this study, all 11 cases of chronic kidney disease in the severe group showed involvement of the right colon. Four out of 11 cases were on hemodialysis. The rate of involvement of right colon in chronic kidney disease with hemodialysis in severe group was 80\% (4/5). Deaths occurred in two of these four cases.

The ECOG performance status score has been known to be closely related to the prognosis of patients with cancer in general. ${ }^{20,21}$ This is easy to use and is universally applied to diseases other than cancer. ${ }^{22-24}$ According to a study on the effect of the ECOG performance status score on the death rate of 3,868 severe patients, ${ }^{25}$ the higher the ECOG performance status score, the higher the hospital mortality was suggesting that increased mortality was not related to the accompanying cancer. In this study, patients with severe ischemic colitis who finally underwent surgery or died had a tendency to have high ECOG performance status score, as verified by the multivariate analysis which showed the ECOG performance status score was an independent factor that could predict severe ischemic colitis.

In conclusion, factors that can predict poor prognosis in ischemic colitis were comorbidity of chronic kidney disease and high ECOG performance status score. Therefore, it is necessary for patients with suspicious ischemic colitis to receive active treatment (e.g., surgery) at an early stage and to be observed carefully if they are treated with chronic kidney disease and if their performance status is poor overall.

\section{CONFLICTS OF INTEREST}

No potential conflict of interest relevant to this article was reported.

\section{REFERENCES}

1. Brandt LJ, Boley SJ. Colonic ischemia. Surg Clin North Am 1992; 72:203-229.

2. Higgins PD, Davis KJ, Laine L. Systematic review: the epidemiology of ischaemic colitis. Aliment Pharmacol Ther 2004;19:729738.

3. Añón R, Boscá MM, Sanchiz V, et al. Factors predicting poor prognosis in ischemic colitis. World J Gastroenterol 2006;12:48754878.

4. Toursarkissian B, Thompson RW. Ischemic colitis. Surg Clin North Am 1997;77:461-470.

5. West BR, Ray JE, Gathright JB. Comparison of transient ischemic colitis with that requiring surgical treatment. Surg Gynecol Obstet 1980;151:366-368.

6. Abel ME, Russell TR. Ischemic colitis: comparison of surgical and nonoperative management. Dis Colon Rectum 1983;26:113-115.

7. Guttormson NL, Bubrick MP. Mortality from ischemic colitis. Dis Colon Rectum 1989;32:469-472.

8. Longo WE, Ballantyne GH, Gusberg RJ. Ischemic colitis: patterns and prognosis. Dis Colon Rectum 1992;35:726-730.

9. Diamond SM, Emmett M, Henrich WL. Bowel infarction as a cause of death in dialysis patients. JAMA 1986;256:2545-2547.

10. Oken MM, Creech RH, Tormey DC, et al. Toxicity and response criteria of the Eastern Cooperative Oncology Group. Am J Clin Oncol 1982;5:649-655.

11. Bower TC. Ischemic colitis. Surg Clin North Am 1993;73:10371053.

12. Brandt LJ, Feuerstadt P, Blaszka MC. Anatomic patterns, patient characteristics, and clinical outcomes in ischemic colitis: a study of 313 cases supported by histology. Am J Gastroenterol 2010;105:2245-2252.

13. Sotiriadis J, Brandt LJ, Behin DS, Southern WN. Ischemic colitis has a worse prognosis when isolated to the right side of the colon. Am J Gastroenterol 2007;102:2247-2252.

14. Medina C, Vilaseca J, Videla S, Fabra R, Armengol-Miro JR, Malagelada JR. Outcome of patients with ischemic colitis: review of fifty-three cases. Dis Colon Rectum 2004;47:180-184.

15. O'Neill S, Yalamarthi S. Systematic review of the management of ischaemic colitis. Colorectal Dis 2012;14:e751-e763.

16. Lim YJ, Son HJ, Kang TW, et al. Clinical patterns and prognostic factors of ischemic colitis. Korean J Gastrointest Endosc 2001;22:76-82.

17. Flobert C, Cellier C, Berger A, et al. Right colonic involvement is associated with severe forms of ischemic colitis and occurs frequently in patients with chronic renal failure requiring hemodialysis. Am J Gastroenterol 2000;95:195-198.

18. Zeier M, Wiesel M, Rambausek M, Ritz E. Non-occlusive mesenteric infarction in dialysis patients: the importance of prevention and early intervention. Nephrol Dial Transplant 1995;10:771-773.

19. Ahn SE, Lee HL, Cho SC, et al. Is end stage renal disease a poor prognosis factor of ischemic colitis? Korean J Gastroenterol 2009; 53:235-238.

20. Buccheri G, Ferrigno D, Tamburini M. Karnofsky and ECOG performance status scoring in lung cancer: a prospective, longitudinal study of 536 patients from a single institution. Eur J Cancer 1996;32A:1135-1141.

21. Corrêa GT, Bandeira GA, Cavalcanti BG, et al. Analysis of ECOG performance status in head and neck squamous cell carcinoma patients: association with sociodemographical and clinical factors, and overall survival. Support Care Cancer 2012;20:2679-2685.

22. Ström K, Boe J. Quality assessment and predictors of survival in long-term domiciliary oxygen therapy. The Swedish Society of Chest Medicine. Eur Respir J 1991;4:50-58.

23. St Julien JB, Aldrich MC, Sheng S, et al. Obesity increases operat- 
766 Gut and Liver, Vol. 9, No. 6, November 2015

ing room time for lobectomy in the society of thoracic surgeons database. Ann Thorac Surg 2012;94:1841-1847.

24. Chamogeorgakis T, Toumpoulis I, Tomos P, et al. External validation of the modified Thoracoscore in a new thoracic surgery program: prediction of in-hospital mortality. Interact Cardiovasc
Thorac Surg 2009;9:463-466.

25. Park CM, Koh Y, Jeon K, et al. Impact of Eastern Cooperative Oncology Group Performance Status on hospital mortality in critically ill patients. J Crit Care 2014;29:409-413. 